\title{
AA DOR - AN ECLIPSING SUBDWARF - BROWN DWARF BINARY
}

\author{
T. Rauch ${ }^{1,2}$
}

AA DOR is an eclipsing, close, post commonenvelope binary (PCEB) consisting of a sdOB primary star and an unseen secondary with an extraordinary small mass $\left(M_{2} \approx 0.066 \mathrm{M}_{\odot}\right)-$ formally a brown dwarf (see Rauch 2004 for details). In a spectral analysis of AA DOR, Rauch (2000) determined $T_{\text {eff }}=42 \mathrm{kK}$ and $\log g=5.2(\mathrm{cgs})$. The determination of the components' masses by comparison of these results with evolutionary models of Driebe et al. (1998) shows a discrepancy to masses derived from radial-velocity and the eclipse curves (Hilditch et al. 2003) $-\log g=\mathbf{5 . 5}$ would be necessary in order to achieve an intersection at $M_{1} \approx 0.330 \mathrm{M}_{\odot}$.

Possible reasons for this discrepancy may be too optimistic error ranges in Rauch (2000) or in the analysis of light curve and radial-velocity curve, or that the evolutionary models of Driebe et al. (1998) are not appropriate in the case of AADOR since these are post-RGB models for non-CE stars.

Since the decrement of the hydrogen Balmer series is a sensitive indicator for $\log g, 107$ highresolution échelle spectra with short exposure times (180 sec) have been taken in Jan 2001 with UVES attached to the ESO VLT. Additional mediumresolution longslit spectra have been taken at the $2.3 \mathrm{~m}$ telescope at SSO in Sept 2003 with the DBS. However, the analysis of both, the UVES spectra (Rauch \& Werner 2003) and the DBS spectra, shows that a $\log g$ higher than 5.2 results in a worse fit to the observation.

Since the secondary is heated by irradiation of the primary up to $\approx 20 \mathrm{kK}$, one can expect a weak $\mathrm{H} \beta$ emission in the UVES spectra. However, we do not find any signature of the secondary. The emission in the line core of $\mathrm{H} \beta$ (Fig. 1) comes clearly from the primary, its phase dependence is likely due to an irradiation effect of the heated secondary on the primary which increases its $T_{\text {eff }}$ by $\approx 7 \mathrm{kK}$, i.e. the primary - taken as an isolated star - would have only $T_{\text {eff }} \approx 35 \mathrm{kK}$, resulting in $\mathrm{a} \approx 10 \%$ smaller mass. A phase-dependent spectral analysis is presently per-

\footnotetext{
${ }^{1}$ Dr.-Remeis-Sternwarte, Sternwartstraße 7, D-96049 Bamberg, Germany (Thomas.Rauch@sternwarte.uni-erlangen.de).

${ }^{2}$ Institut für Astronomie und Astrophysik, Sand 1, D72076 Tübingen, Germany.
}

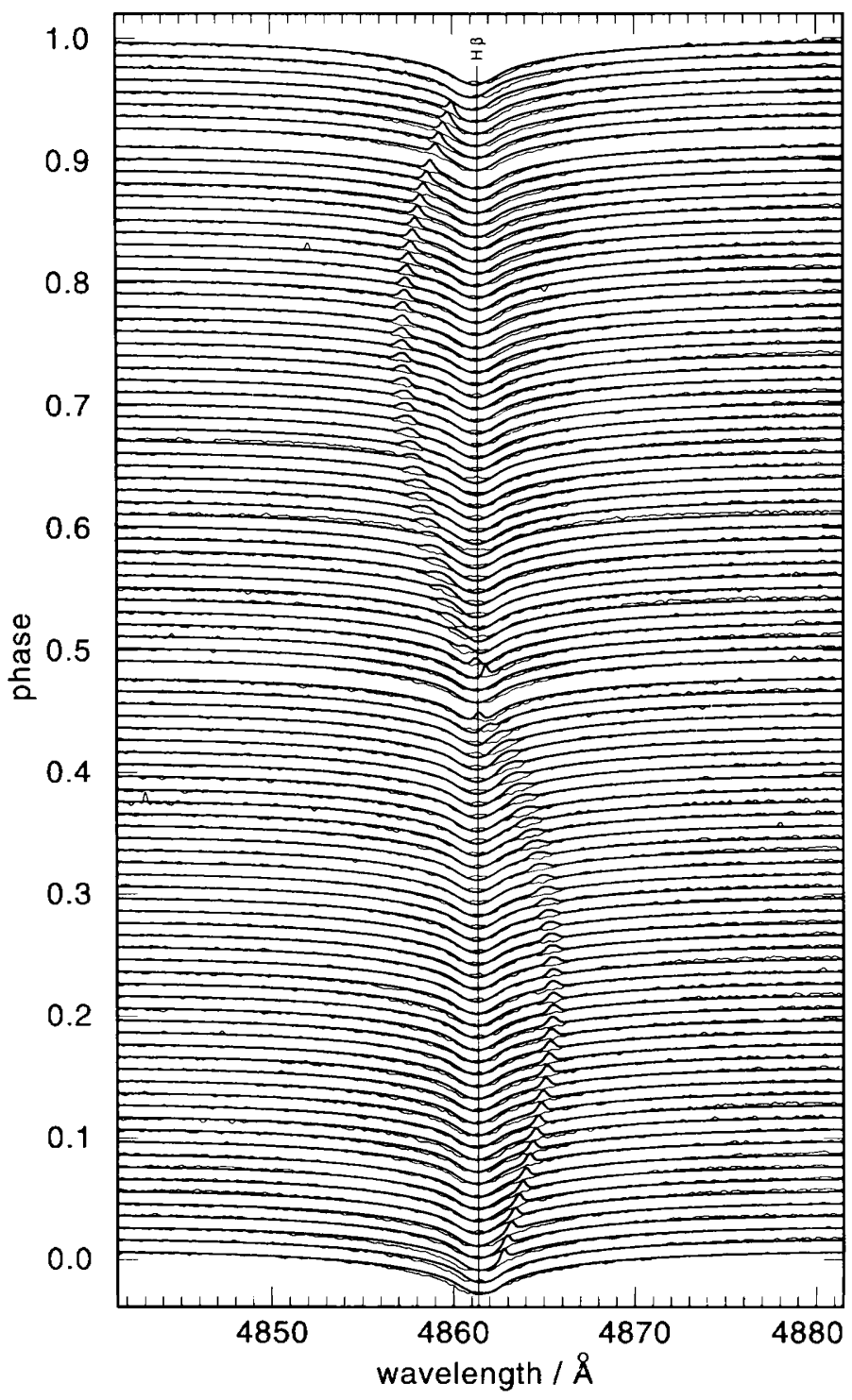

Fig. 1. Section of the UVES spectra around $\mathrm{H} \beta$ compared with synthetic spectra. A weak $\mathrm{H} \beta$ emission is used to represent the secondary's radiation.

formed in order to investigate on this effect. It appears possible that this is one of the main reasons for the disagreement in the mass-radius relation.

\section{REFERENCES}

Driebe, T., Schönberner, D., Blöcker, T., \& Herwig, F. 1998, A\&A, 339, 129

Hilditch, R.W., Kilkenny, D., \& Lynas-Gray, A.E., Hill, G. 2003, MNRAS, 344, 644

Rauch, T. 2000, A\&A, 356, 665

Rauch, T. 2004, Ap\&SS, in press

Rauch, T., \& Werner, K. 2003, A\&A, 400, 271 